\title{
Transcaval approach for embolization of type II Endoleak following endovascular aortic aneurysm repair
}

\author{
Eddie Hyatt ${ }^{*} \mathbb{D}$, Joseph N. McLaughlin, Hriday Shah and Sanjeeva P. Kalva
}

\begin{abstract}
Type II endoleak is a common complication following endovascular aortic aneurysm repair and can lead to an increased risk of aneurysmal expansion and rupture. The most frequently employed strategies to treat Type II endoleak involves catheterization of the branch vessels responsible for the endoleak or accessing the aneurysm sac through a percutaneous approach. An endovascular transcaval approach for embolization of the aneurysmal sac provides an alternate strategy with comparable success rates. This technique is advantageous when the endoleak is predominantly on the right side of the aneurysm sac and/or when a direct access to the aneurysm sac through a percutaneous approach is not feasible.
\end{abstract}

Keywords: Abdominal aortic aneurysm, Endovascular aneurysm repair, Embolization, Transcaval embolization, Endoleak, Type II endoleak

\section{Background}

Endovascular abdominal aortic aneurysm repair (EVAR) is the standard of care for the treatment of most aneurysms greater than $5.5 \mathrm{~cm}$ diameter (Yang et al., 2016). Unfortunately, type 2 endoleak (T2E) is a common and often unavoidable complication resulting from incomplete exclusion of the aneurysm sac from the circulation via retrograde flow from branches of the abdominal aorta which can lead to aneurysm expansion and ultimately rupture.

Currently there is no consensus as to the optimal treatment strategy for T2E. The indication for treatment typically includes persistent endoleak $(>6$ months) and continued sac expansion $(>0.5 \mathrm{~cm})$ (Yamada et al., 2015; Ozdemir et al., 2013). Frequently used strategies include: 1) transarterial embolization of the feeding vessel accessed from the superior mesenteric or internal iliac artery collaterals; and 2) embolization via direct sac puncture from a translumbar (Ozdemir et al., 2013) or transabdominal (Zener et al., 2018) approach. The surgical options include ligation of the source vessels laparoscopically or open repair with feeding vessel ligation and aneurysmorraphy (Scali et al., 2013).

Transcaval embolization (TCE) provides an additional strategy with comparable success rates (Yang et al., 2016) while avoiding some limitations and reducing some risks inherent to other techniques, including technical failure due to inability to cannulate the feeding vessel, inadvertent injury to periaortic structures by traversing multiple tissue planes, prone patient positioning, and potential need for general anesthesia.

\section{Transcaval approach for Endoleak embolization Technique}

Review of prior imaging is essential for optimal patient selection and procedure planning. The aneurysm sac ideally abuts the IVC and contains a minimal amount of wall calcification. From the bilateral groins or groin and neck an intravascular ultrasound (IVUS) probe is used to direct the tip of a vascular sheath against the inferior vena cava (IVC) wall near the site of the T2E

\footnotetext{
* Correspondence: Eddie.Hyatt@utsouthwestern.edu

Division of Vascular and Interventional Radiology, Department of Radiology,

University of Texas Southwestern Medical Center, 5323 Harry Hines

Boulevard, Dallas, TX 75390, USA
} 


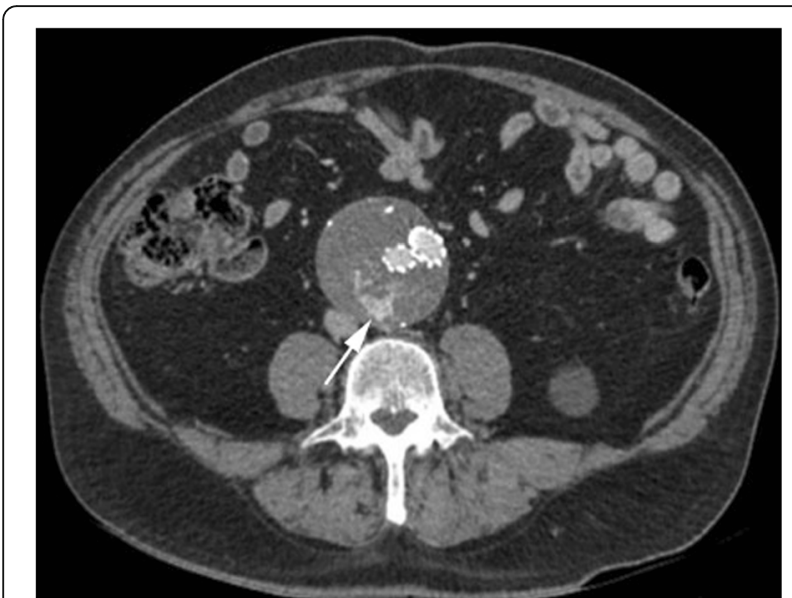

Fig. 1 Axial computed tomography angiography (CTA) through the lower abdomen shows an abdominal aortic aneurysm with a T2E (white arrow) arising from an adjacent lumbar artery

identified on IVUS as patent cavities or inflow vessel tracts. The aneurysm sac is then punctured with a needle through the sheath using fluoroscopy and/or IVUS. Access of the endoleak cavity is confirmed by injection of dye. Wire and catheter are used to further select the endoleak cavity as needed. A microcatheter is eventually placed into the aneurysm sac and the endoleak cavity is embolized with coils, liquid embolics, or both.

\section{Case}

A 75-year-old man underwent EVAR for a $6.1 \mathrm{~cm}$ abdominal aortic aneurysm. On follow-up CT angiography
(CTA) imaging obtained 9 months later the aneurysm measured $6.8 \mathrm{~cm}$ and a T2E was seen arising from a lumbar artery (Fig. 1). Given the persistent T2E and continued enlargement of the aneurysm the decision to treat was made.

The procedure was performed under conscious sedation. The common femoral veins were accessed bilaterally with micropuncture sets (Vascular Solutions, Inc., Minneapolis, MN). The right femoral vein micropuncture sheath was exchanged for a Rosch-Uchida transjugular liver access set (Cook Medical, LLC, Bloomington, IN). The left femoral micropuncture sheath was exchange for a $9 \mathrm{~F}$ vascular sheath through which an IVUS probe (Volcano Corporation, San Diego, CA) was advanced into the IVC.

Under fluoroscopic and IVUS guidance the aneurysm sac was accessed near the endoleak with the Rosch-Uchida liver access set (Fig. 2). The inner needle was removed, and contrast was injected through the catheter confirming correct positioning within the aneurysm sac. This straight catheter was exchanged over the wire for a $5 \mathrm{~F}$ angle tipped catheter (Terumo Medical Corporation, Somerset, NJ) which was used to select the endoleak cavity. A Progreat microcatheter/ microwire set (Terumo Medical Corporation, Somerset, NJ) was advanced through the catheter into the aneurysm sac and position was confirmed with contrast injection.

Next, the microcatheter was flushed with $5 \%$ dextrose solution. Ethylene vinyl alcohol liquid embolic (Onyx $\left.{ }^{\circ} 18\right)$ (Micro Therapeutics, Inc., Irvine, CA) was
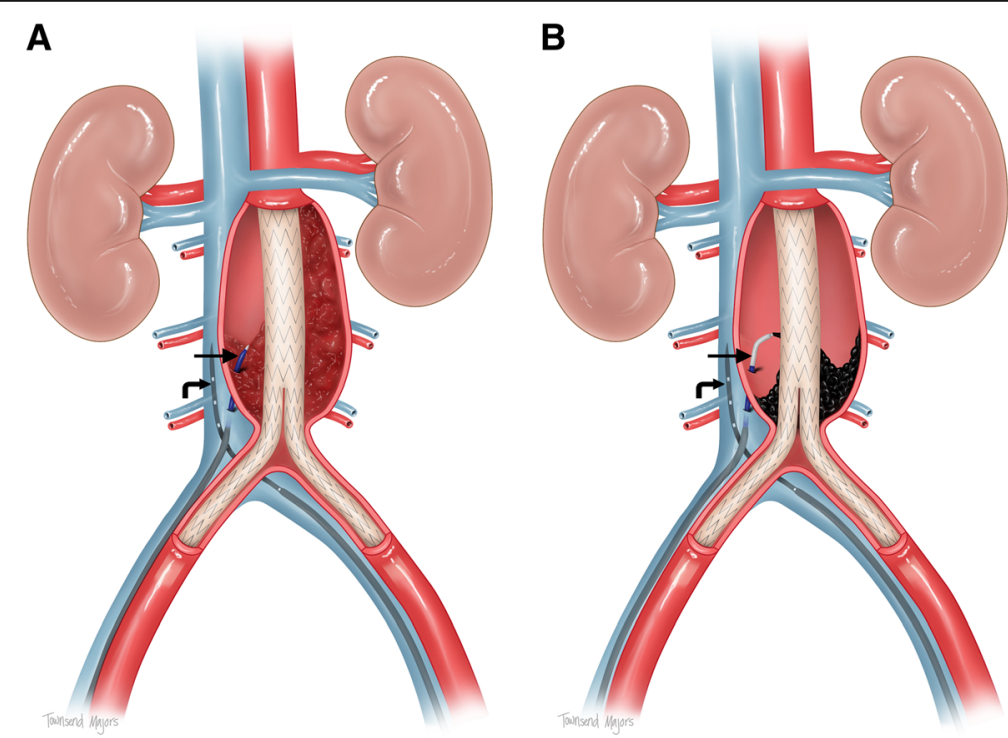

Fig. 2 a. Rosch-Uchida liver access set (straight arrow) is used to access the aortic aneurysm sac. The aneurysm sac is accessed, near the site of the type II endoleak, from the inferior vena cava (IVC) under sonographic guidance with an intravascular ultrasound (IVUS) probe (curved arrow). b. The Rosch-Uchida needle was exchanged for an angle-tipped catheter and microcatheter (straight arrow). Embolic material is administered via the microcatheter. IVUS probe (curved arrow) 
then administered through the microcatheter into the aneurysm sac, in the region of the T2E, under sonographic and fluoroscopic guidance (Figs. 2 and 3). The embolization was terminated once the endoleak was no longer visualized with IVUS. The catheters and IVUS probe were removed. Completion cavogram was performed through the femoral vein sheaths. Femoral vein sheaths were then removed, and hemostasis was achieved at the venotomy sites with manual compression. Total procedure time was $2.5 \mathrm{~h}$. Follow up imaging (Fig. 4) demonstrated good radiographic results.

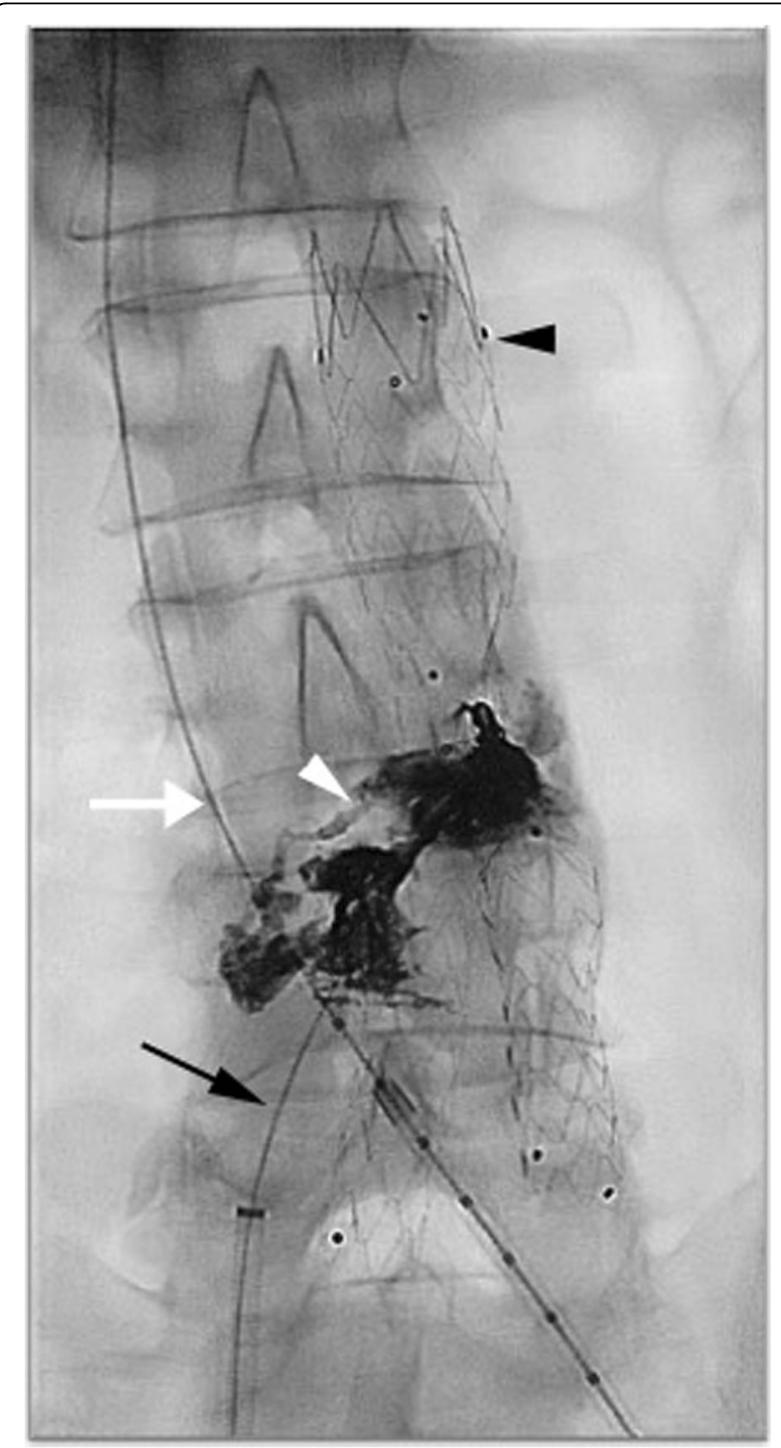

Fig. 3 Fluoroscopic image obtained during the embolization procedure showing ethylene vinyl alcohol liquid embolic (Ony $x^{\circledast}$ ) administration via the microcatheter (black arrow). Guidewire and intravascular ultrasound (IVUS) probe (white arrow) within the IVC inserted through the left common iliac vein. Aortic stent graft (black arrowhead). Onyx ${ }^{\circledast}$ within the excluded aneurysm sac (white arrowhead)

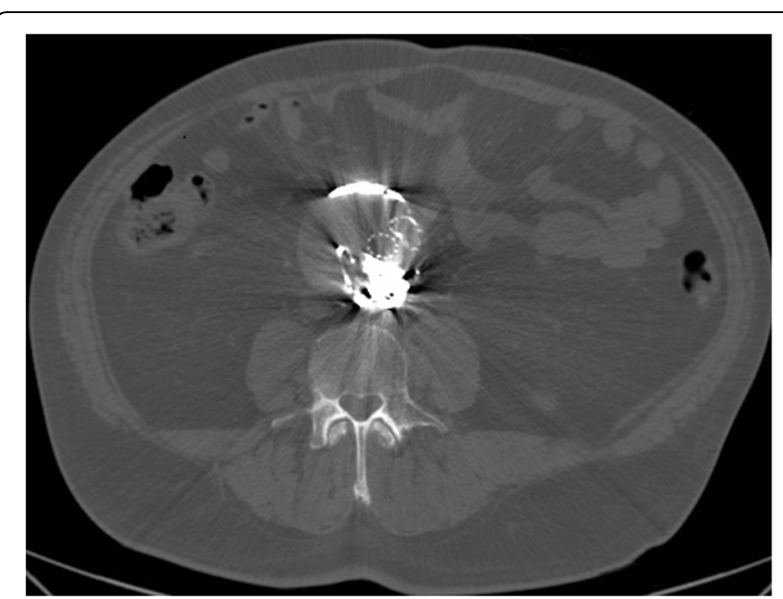

Fig. 4 Post-embolization axial CTA through the lower abdomen at the same level as image in Fig. 1 shows embolic material filling the portion of the aneurysmal sac which previously demonstrated endoleak

\section{Enhanced techniques}

Real-time visualization of the access needle using "side-firing" IVUS probes is invaluable when performing TCE. First, it allows the endoleak cavity to be directly targeted. Targeted embolization of the endoleak cavity decreases the pressure within the aneurysm, increasing the likelihood of treatment success. Cessation of color Doppler flow on IVUS also determines the endpoint of embolization (Thakrar et al., 2013). (Alternatively, embolization can be stopped once intra-sac pressure stabilizes or has decreased by $50 \mathrm{mmHg}$ (Gandini et al., 2014).) Appropriate endpoint determination decreases the risk of nontargeted embolization within the cava and/or pulmonary embolism. Use of IVUS also decreases the risk of creation of type III endoleak from unintentional puncture of the endograft, injury to periaortic/caval structures, aortocaval fistula formation, and retroperitoneal hemorrhage.

In thin patients, transabdominal ultrasound may work equally well. Post embolization, endoleak occlusion can also be confirmed in the angiosuite via realtime contrast enhanced US.

\section{Conclusion}

T2E is a common, often unavoidable complication of EVAR which can lead to an increased risk of aneurysmal expansion and rupture. TCE is technically less demanding and faster (requiring less fluoroscopy time) approach to managing T2E. We believe TCE can potentially have a higher technical success rate and a decreased risk profile when compared with other more common catheterization techniques. Operators may consider TCE as a feasible first line alternative approach in the management of T2E. 


\section{Abbreviations}

AAA: Abdominal aortic aneurysm; CT: Computed tomography;

CTA: Computed tomography angiography; EVAR: Endovascular abdominal aortic aneurysm repair; IVC: Inferior vena cava; IVUS: Intravascular ultrasound;

T2E: Type II endoleak; TCE: Transcaval embolization

\section{Acknowledgements}

Townsend Majors, M.A. Medical Illustrator.

\section{Funding}

N/A

\section{Availability of data and materials}

N/A

\section{Authors' contributions}

EH and JM drafted manuscript. SK contributed to concept and design and provided guidance throughout the project and made revisions. HS reviewed manuscript and made revisions. All authors read and approved the final manuscript.

\section{Ethics approval and consent to participate}

N/A. According to our institutional policy manual case reports are a category of work that generally do not require IRB approval.

\section{Consent for publication}

N/A

\section{Competing interests}

The authors declare that they have no competing interests.

\section{Publisher's Note}

Springer Nature remains neutral with regard to jurisdictional claims in published maps and institutional affiliations.

Received: 4 September 2018 Accepted: 27 December 2018

Published online: 09 January 2019

\section{References}

Gandini R, Chiocchi M, Loreni G et al (2014) Treatment of type II endoleak after endovascular aneurysm repair: the role of selective vs. nonselective transcaval embolization. J Endovasc Ther 21(5):714-722

Ozdemir BA, Chung R, Benson RA et al (2013) Embolisation of type 2 endoleaks after endovascular aneurysm repair. J Cardiovasc Surg 54(4):485-490

Scali ST, Vlada A, Chang CK, Beck AW (2013) Transcaval embolization as an alternative technique for the treatment of type II endoleak after endovascular aortic aneurysm repair. J Vasc Surg 57(3):869-874

Thakrar PD, Petersen BD, Kaufman JA (2013) Intravascular ultrasound for transvenous interventions. Tech Vasc Interv Radiol 16(3):161-167

Yamada M, Takahashi H, Tauchi Y, Satoh H, Matsuda H (2015) Open surgical repair can be one option for the treatment of persistent type II Endoleak after EVAR. Ann Vasc Dis 8(3):210-214

Yang RY, Tan KT, Beecroft JR, Rajan DK, Jaskolka JD (2016) Direct sac puncture versus transarterial embolization of type II endoleaks: an evaluation and comparison of outcomes. Vascular 25(3):227-233

Zener R, Oreopoulos G, Beecroft R, Rajan DK, Jaskolka J, Tan KT (2018) Transabdominal direct sac puncture embolization of type II Endoleaks after endovascular abdominal aortic aneurysm repair. J Vasc Interv Radiol 29(8): $1167-1173$

\section{Submit your manuscript to a SpringerOpen ${ }^{\circ}$ journal and benefit from:}

- Convenient online submission

- Rigorous peer review

- Open access: articles freely available online

High visibility within the field

- Retaining the copyright to your article 